\title{
Atrial mechanical hypofunction after electrical cardioversion of persistent or long-lasting persistent atrial fibrillation: a retrospective cohort study
}

\author{
Renato De Vecchis, ${ }^{1}$ Andrea Paccone ${ }^{2}$, Marco Di Maio ${ }^{3}$ \\ ${ }^{1}$ Medical and Polyspecialist Centre, "S. Gennaro dei Poveri Hospital", Naples; ${ }^{2}$ Department of Cardiology, University \\ of Bari “Aldo Moro; ${ }^{3}$ Department of Cardiology, University of Campania” Luigi Vanvitelli”, Naples, Italy
}

\begin{abstract}
In the present retrospective cohort study we have evaluated the missed or delayed atrial mechanical recovery in a population of patients with persistent or long-lasting persistent atrial fibrillation (AF) who achieved restoration of sinus rhythm on the ECG by electrical cardioversion (ECV). The endpoint of our study was the failure to recover the normal mechanics of the left atrium. Inclusion criterion was the persistent or long-lasting persistent atrial fibrillation successfully treated by means of ECV, provided that a pertinent documentation was made available, comprising ECG, conventional 2D echo-color-Doppler and speckle tracking echocardiography (STE) evaluation, with also a STE assessment of the atria at the days 1, 30 and 90 from the ECV freely available
\end{abstract}

Correspondence: Renato De Vecchis, Medical and Polyspecialist Centre, DSB 29 "S. Gennaro dei Poveri Hospital", via S. Gennaro dei Poveri 25, 80136 Napoli, Italy.

Tel.+39.081.7516932 - Mobile: +39.348 .3313530 .

E-mail: devecchis.erre@virgilio.it

Key words: Atrial fibrillation; electrical cardioversion; atrial hypofunction.

Financial disclosure: The authors declare that the present article did not receive any source of funding.

Conflict of interest: The authors do not have any conflicts of interest to declare.

Informed consent: not applicable.

Contributions: RDV, AP, study concept and design; RDV, contribution to data curation; RDV, AP, MDM manuscript drafting, review and editing. All the authors have read and approved the finale version of the manuscript.

Received for publication: 3 October 2019.

Accepted for publication: 24 November 2019.

COpyright: the Author(s), 2019

Licensee PAGEPress, Italy

Monaldi Archives for Chest Disease 2019; 89:1178

doi: 10.4081/monaldi.2019.1178

This article is distributed under the terms of the Creative Commons Attribution Noncommercial License (by-nc 4.0) which permits any noncommercial use, distribution, and reproduction in any medium, provided the original author(s) and source are credited. within the clinical record of the patient. Out of a total of 80 patients with persistent or long-standing persistent AF, retrospectively enrolled, as many as $22.5 \%$ of them did not achieve the normalization of their atrial STE profile, even though they had been converted to sinus rhythm on the ECG by means of ECV. The building of ROC curves allowed us to establish that early measurements of global atrial strain could serve to predict both the risk of failure to recover the atrial mechanical function and the one of AF relapses over a 12-month follow-up. The values of $18 \%$ and $17 \%$ were also calculated to serve as cut off values, respectively, for the risk of atrial mechanical dysfunction and for the risk of AF relapses over a 12-month follow-up. Failure to recover the atrial reservoir function can accompany a restoration of sinus rhythm on the ECG in patients with long-standing persistent AF. In this case a serial STE evaluation could be useful to evaluate the atrial hypofunction over time.

\section{Introduction}

The evaluation by speckle tracking echocardiography (STE) of patients who have recently performed an electrical cardioversion (ECV) for atrial fibrillation (AF) is an approach that emphasizes appropriately the role of this echocardiographic method in the AF management. If atrial fibrillation has lasted for a long time, for more than $48 \mathrm{~h}$, persistent $\mathrm{AF}$, or for more than twelve months, long-lasting persistent $\mathrm{AF}$, the method adopted for converting $\mathrm{AF}$ to sinus rhythm is usually the ECV, although, in the most of the dedicated centers, an initial attempt at pharmacological cardioversion with the use of intravenous amiodarone or propafenone is generally implemented [1]. Unlike recent onset atrial fibrillation ( $\leq 48 \mathrm{~h}$, paroxysmal AF), the persistent or long-lasting persistent AF requires a minimum period of three weeks of full anticoagulation, carried out by means of warfarin, in the case of valvular AF, i.e., associated with mitral stenosis or mitral prosthesis, or, in the case of non-valvular AF, carried out by means of a non-vitamin $\mathrm{K}$ antagonist oral anticoagulant.

A not completely clarified issue is the fact that a post-cardioversion very prolonged stunning might take place in a certain number of patients. In the case of persistent or long-lasting persistent AF, it is well-known that an atrial both anatomic and electrical remodeling takes place usually. Moreover, in the patients converted to sinus rhythm, it is known that a certain timeframe has to pass before this unfavorable remodeling can regress or disappear.

In short, in the period immediately following the ECV, a reduction of global atrial strain $(\mathrm{GSA}+)$ in the reservoir phase of the atrial cardiac cycle is detected usually using STE. The acronym adopted is GSA+, which stands for global strain of the 
left atrium, while the sign + represents the percentage increase in left atrial volume that occurs at this stage. In patients with persistent AF or long- lasting persistent AF, low values of GSA+, detected in the hours immediately subsequent to the $\mathrm{ECV}$, are a relatively frequent finding [2]. Moreover, in about $75 \%$ of patients, there is a recovery of the atrial global strain over the course of the days, with consequent restoration of the end-diastolic contractile efficiency of the atria, and normalization of their function of mechanical contribution to the ventricular filling. However, in about a quarter of patients with previous persistent or long-lasting persistent atrial fibrillation converted to sinus rhythm, the pharmacological or electrical cardioversion is not able to gain the atrial mechanical recovery, even if sinus rhythm has been achieved on ECG [3]. The due emphasis should be given to this ominous functional behavior, because of its unfavorable prognostic implications. The lack of atrial mechanical normalization can be diagnosed on the basis of the Doppler-velocimetric profile of the mitral valve using the echo 2D Doppler or by relying on the STE, namely taking into account the GSA+ [4]. Persistent low values of GSA+ are irreconcilable with a good recovery of the atria. Even when not affected by recurrences of atrial fibrillation, the atria may be more vulnerable to the risk of blood stasis, red cell stacking and left atrial appendage thrombosis. Furthermore, in these cases the emptying velocity of the left atrial appendage is lower. Besides, more often the atria which do not recover, show increased volume and are associated with high circulating levels of natriuretic peptides (atrial natriuretic peptide and B-type natriuretic peptide), an indirect proof of high left ventricular end-diastolic pressure. It is also ascertained that if the atria are involved by a prolonged or permanent "stunning", with a low GSA+, they are likely to be burdened by a greater amount of fibrosis. Both atrial fibrosis and high ventricular enddiastolic pressure, the latter testified by a high E / e' ratio [5], not only contribute to weakening or suppressing atrial mechanical contraction, even in the presence of sinus rhythm on ECG, but are risk factors for new episodes of atrial fibrillation. Another potential implication could be that in these patients devoid of effective atrial contribute, though showing sinus rhythm on ECG, a reduced GSA+ value could strengthen the indication for loop recorder implantation, to detect possible atrial arrhythmias, and/or for anticoagulation therapy, to prevent cardioembolic events [6]. Alternatively, patients without atrial mechanical recovery, identified by means of STE, could usefully undergo pharmacological prophylaxis with amiodarone or other antiarrhythmic agents against AF relapses [7]. On this point, however, the experimental and clinical evidence is sparse or much debatable $[6,7]$.

\section{Aims of the study}

The studies regarding the topic of the relationship between recurrent atrial fibrillation and global left atrial strain (GSA + ) are relatively few and all characterized by rather small sample sizes. Therefore, we felt that there was still enough room to perform an assessment of diagnostic odds ratio (DOR) concerning the diagnostic accuracy of the possible values of left atrial strain measured immediately after ECV ( $3 \mathrm{~h}$ later) to be used as predictors of the risk of failure to recover the atrial function within the subsequent follow-up period of 12 months.

Furthermore, paying attention to the values of global left atrial strain $(\mathrm{GSA}+)$ measured at the third hour from ECV, we searched for the cut-off value capable of better discriminating the patients who would have developed AF relapses from those who would have remained free from $\mathrm{AF}$ in the subsequent 12-month follow-up period.

\section{Methods}

The endpoint of our retrospective cohort study was the failure to recover the normal mechanics of the left atrium. Inclusion criteria were the persistent or long-lasting persistent atrial fibrillation successfully treated with ECV, provided that a pertinent documentation was made available, comprising ECG, conventional 2Dechocolor-Doppler and STE evaluation, with also a STE assessment of the atria at the days 1, 30 and 90 freely available within the clinical record of the patient. For this study the Institutional Review Board Approval was not required, considering the study features (retrospective cohort study). Moreover, this study was conducted in compliance with the ethical standards of the responsible institution on human subjects as well as with the Helsinki Declaration. This study did not involve experiments on animals.

As regards the origin of the data used for making comparisons, it is composite, due to the fact that the related database collects contributions attributable to patients belonging to the Cardiology Outpatient Units and Inpatient Divisions of the ASL Napoli 1 Centro (Naples, Italy), as well as to the Arrhythmology Operational Unit of the "Pineta Grande" Clinic of Castelvolturno (CE), Italy.

\section{Equipment}

\section{Conventional echocardiography evaluation}

All the echocardiograms were performed by the same cardiologist (RDV) with specific training and experience in cardiovascular echocardiography, by using the portable Philips Sparq ultrasound machine with a $2.5 \mathrm{mHz}$ transducer. All measurements were performed according to the Recommendations of the American Society of Echocardiography and the European Association of Cardiovascular Imaging [8].

In the subsequent analysis the following parameters were considered: left atrium indexed volume (LAVi), left ventricle ejection fraction (LVEF) and the ratio of early diastolic mitral inflow velocity to early diastolic myocardial relaxation velocity (average E/e' ratio), considered a marker of diastolic ventricular function.

\section{Speckle tracking echocardiography}

The global and regional LA myocardial strain was measured by 2DSTE. Grey scale imaging of the four chamber and two-chamber views was obtained with a frame rate of $60-80 \mathrm{~Hz}$. Recordings were processed with acoustic-tracking software (EchoPAC, GE Healthcare, Chicago, IL, USA), allowing off-line semi-automated speckle-based strain analysis. Briefly, lines were manually traced along the LA endocardium. An additional epicardial line was automatically generated by software, creating a region of interest (ROI). After manually adjusting the ROI shape, the software divided the LA region into six segments and generated the longitudinal strain curves. Once the longitudinal atrial strain curves were obtained, the following measurements were performed: positive global atrial strain (GSA+), during the reservoir phase, plotted as a positive curve at the aortic valve closure and negative global atrial strain (GSA-), during the left atrial systole, plotted as a negative curve with a peak after the $\mathrm{P}$ wave on ECG. 
For the planned analysis GSA+ was considered, because it has been recognized to be the most important index of left atrial mechanical function [9]. All data were digitally stored and analyzed off-line with customized software.

\section{Statistical analysis}

All statistical tests were performed with a commercially available statistical analysis program (SPSS 15.0 for Windows, SPSS Inc., Chicago, IL, USA). The distribution of the data was assessed using the one-sample D'Agostino-Pearson test. Continuous variables displaying normal distribution were expressed as mean \pm $\mathrm{SD}$, while values with asymmetric distribution were expressed as medians with interquartile ranges. Categorical variables were presented as $\%$.

One-way ANOVA test was used, in order to generate the pertinent graph, showing the splitting of the values of GSA+, measured after three hours from ECV, across the three identified categories: i) failure to recover the atrial function; ii) relatively early restoration of the atrial reservoir function- detected from one week to one month after the ECV; iii) relatively late restoration of the atrial reservoir function, identified not sooner than three months from the ECV. Besides, two Receiver Operating Characteristic (ROC) curves were built. The first ROC curve was built to identify among the GSA+ values taken 1 to $3 \mathrm{~h}$ after the ECV the most accurate cut-off value capable of discriminating with the best balance of sensitivity and specificity the patients who would have gained the atrial mechanical recovery from those unable to retrieve the atrial mechanical function during a 3-month follow-up. Additionally, a second ROC curve was planned concerning the GSA+ values taken 1 to $3 \mathrm{~h}$ after the ECV in order to detect the best cut-off value able to separate the patients who would have suffered from AF relapses from those who would have remained free from AF recurrences during a 12-month follow-up.

\section{Results}

Patients who did not achieve the normalization of the atrial reservoir phase of the atrial mechanical cycle were $18 / 80(22.5 \%)$. Their mean GSA+ measured from one to three hours after ECV was $16.44 \pm 4 \%$. On the contrary, patients whose left atrial mechanical recovery was identified from one week to one month after cardioversion were $37 / 80$ (46.2\%). Their mean GSA+ measured from one to three hours after ECV was $22.45 \pm 3 \%$. Moreover, patients whose left atrial recovery was identified at measurements performed three months after cardioversion were 25/80 (31.2\%). Their mean GSA+ measured from one to three hours after ECV was $20.20 \pm 2.82 \%$ (Figure 1). The patients who experienced one or

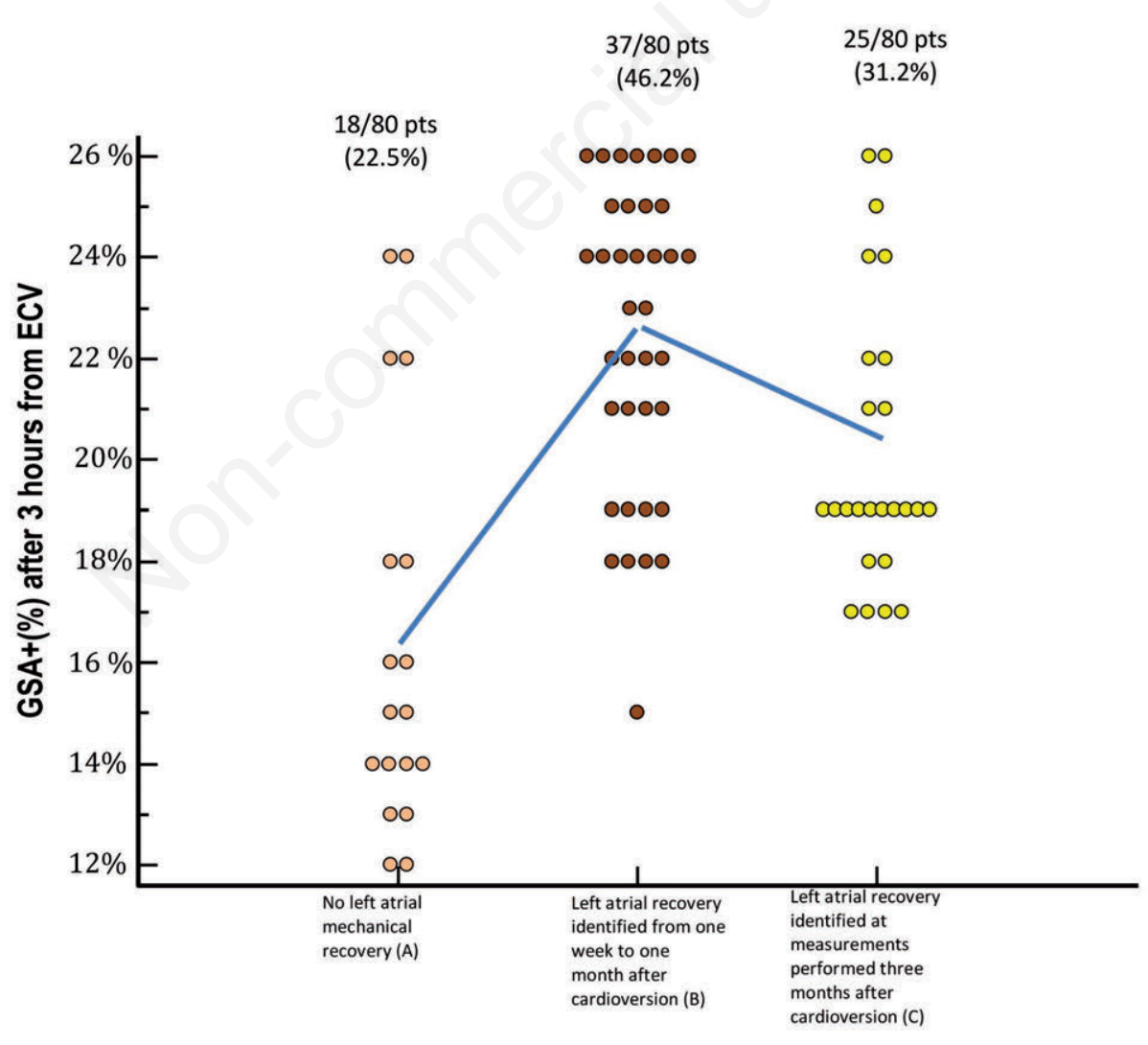

Figure 1. In this plot the global left atrial strain (GSA+) values after $3 \mathrm{~h}$ from the ECV are represented with regard to their distribution across the 3 categories corresponding to the different behavior in the short-medium term of the left atrial reservoir function: failure to recover the atrial function (GSA+ $<18 \%$ ) still persisting after 3 months (A); relatively early restoration of the atrial reservoir function $(G S A+>18 \%)$, detected from one week to one month after the ECV (B); relatively late restoration of the atrial reservoir function, identified not sooner than three months from the ECV (C). The graph provides a visual representation of the fact that low GSA+ values at the third hour are associated with increased probability of post-cardioversion failure to recover the reservoir function of the left atrium. The blue connecting lines conjoin the mean values of GSA+ of the three groups. pts, patients; ECV, electrical cardioversion. 
more AF relapses were 19/80 (23.75\%) over a 12-month follow-up (Figure 2). The mean GSA+ value after 3 hours from ECV in patients without AF recurrences $(n=61)$ was $21.85 \pm 3.09 \%$ (mean $\pm \mathrm{SD}$ ) while the corresponding value in patients with $\mathrm{AF}$ recurrences $(n=19)$ was $15.74 \pm 2.47 \% \quad($ mean $\pm S D)$. Thus, the GSA + was significantly higher $(\mathrm{p}<0.001$ using ANOVA) in the group of patients without AF recurrences compared to the one of patients experiencing one or more AF relapses during the observation period of 12 months (Figure 2).

Based on the results of the analysis of the ROC curves, the value of "basal" global atrial strain (GSA+), that is the GSA+ measured 1 to $3 \mathrm{~h}$ after ECV, was associated with a significantly decreased risk of failure to recover the atrial reservoir function in the event that it was higher than $+18 \%$ (sensitivity: $83.2 \%$; specificity: 76.7\%) (Figure 3). Likewise, the value of "basal" global atrial strain (GSA+), that is the GSA+ measured 1 to $3 \mathrm{~h}$ after ECV, was associated with a significantly decreased risk of AF relapse over a follow-up of 12 months in the event that it was higher than $+17 \%$ (sensitivity: $73.7 \%$; specificity: $95.1 \%$ ) (Figure 4 ). In addition, in Figure 1 the global left atrial strain (GSA+) values measured 1 to 3 hours after the ECV are represented with regard to their distribution across the 3 categories corresponding to the different behavior in the short-medium term of the left atrial reservoir function: failure to recover the atrial function (GSA $+<18 \%$ ) still persisting after 3 months (A); relatively early restoration of the atrial reservoir function $(\mathrm{GSA}+>18 \%)$, detected from one week to one month after the ECV (B); relatively late restoration of the atrial reservoir function, identified not sooner than three months from the ECV (C). The graph provides a visual representation of the fact that low GSA+ values at the third hour are associated with increased probability of post- cardioversion failure to recover the reservoir function of the left atrium.

\section{Discussion}

Our study has outlined the problem of recovering atrial efficiency after electrical cardioversion. Currently the increasing use of radiofrequency ablative therapy for AF has raised some doubts especially in the case of ablation applied to persistent or longlasting persistent $\mathrm{AF}$ in patients with heart failure with reduced ejection fraction (HFREF) [10]. Moreover, the CABANA trial [11], that used the composite end point of all cause-death, disabling stroke, major bleeding events and cardiac arrest, was not able to document with the method of the intention to treat a superiority of trans-catheter ablation compared to conventional therapy possibly integrated by the addition of antiarrhythmic drugs. Our study in turn raises the question that in persistent or longlasting persistent AF the rhythm control strategy could be ineffective because in about a quarter of cases it would bring about a fictitious improvement, i.e., the recovery of sinus rhythm on $\mathrm{ECG}$, which does not find a mechanical equivalent due to a real atrial electromechanical dissociation. In other words, the ECG finding of sinus rhythm does not yield the benefits that such a change should entail as the reservoir phase of the mechanical atrial cycle is indefinitely impaired or suppressed. Therefore also the end-diastolic contribution to ventricular filling is abolished in these cases. Relatively few studies have dealt with the subject $[2,3,6,12,13]$. However, electrocardiographic success is not syn-

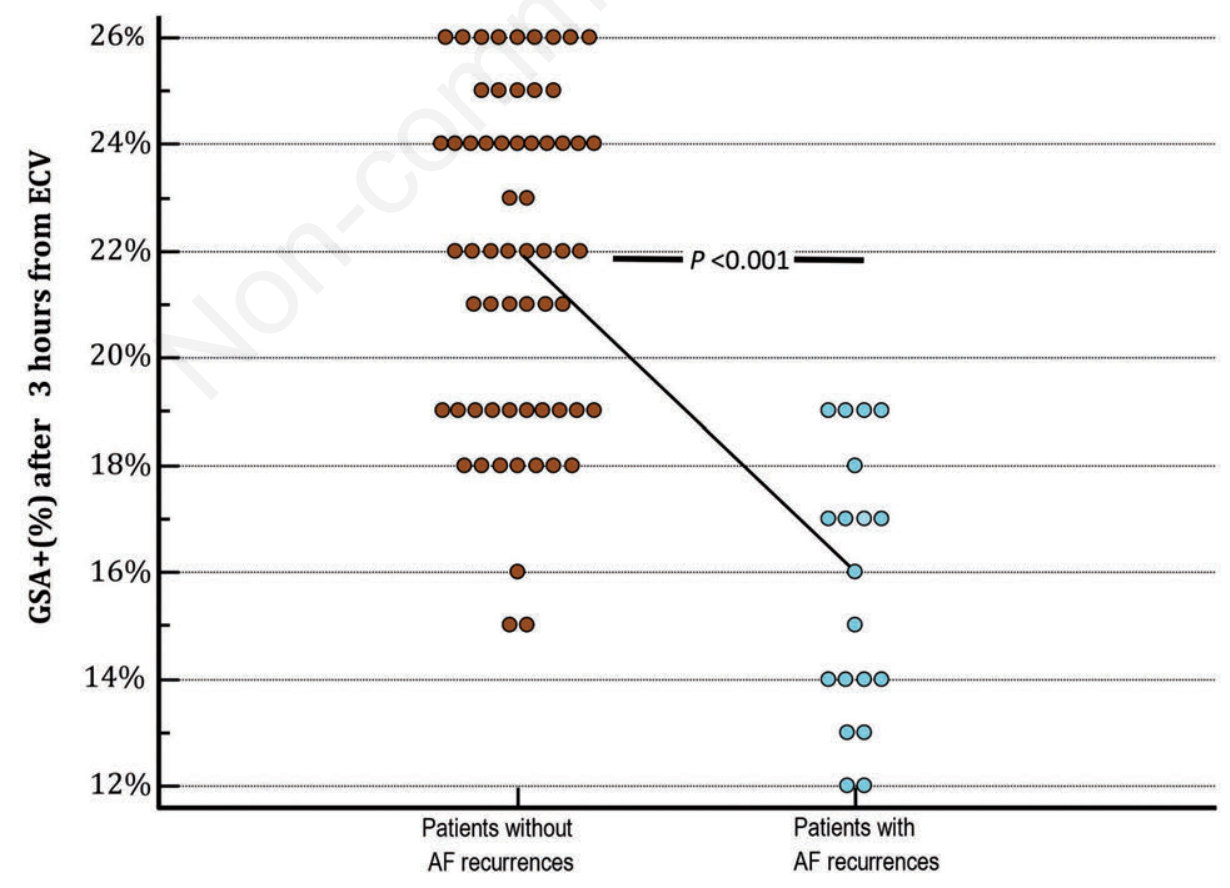

Figure 2. The mean GSA+ value detected after 3 hours from ECV is significantly higher ( $<<0.001$ using ANOVA) in the group (no.61) of patients without $A F$ recurrences compared to the one $(n=19)$ of patients experiencing one or more $A F$ relapses during an observation period of 12 months; GSA+ value after 3 hours from ECV (mean \pm SD) in patients without AF recurrences: $+21.85 \pm 3.09 \%$; GSA + mean value after 3 hours from ECV (mean $\pm \mathrm{SD}$ ) in patients with $\mathrm{AF}$ recurrences: $+15.74 \pm 2.47 \%$. GSA+, global strain of the left atrium; $\mathrm{AF}$, atrial fibrillation; ECV, electrical cardioversion; ANOVA, analysis of variance; pts, patients; st. dev., standard deviation. 


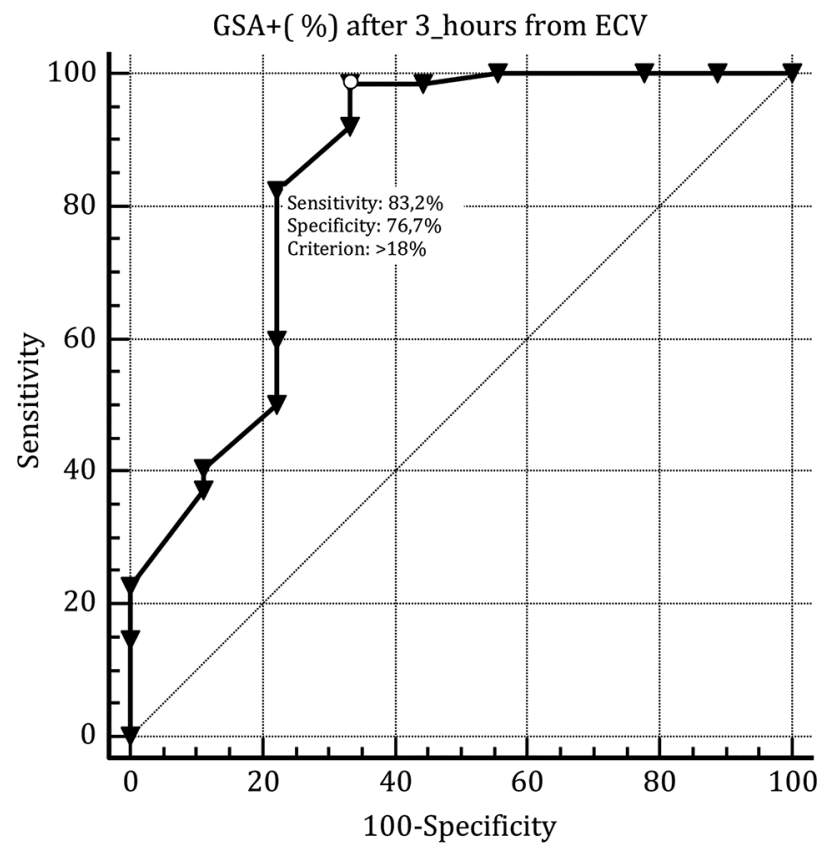

Figure 3. The value of "basal" global atrial strain (GSA+), that is the GSA+ measured after 3 hours from electrical cardioversion is associated with a significantly decreased risk of failure to recover the left atrial reservoir function when it is higher than to the cutoff value of $18 \%$.

onymous of mechanical and/or clinical success. Surveillance should not be limited to the prescription of anticoagulant therapy for 4 weeks after cardioversion. It should also include the serial STE ultrasound practice to evaluate the evolution over time of the post-procedural residual atrial mechanical hypofunction. There are patients whose atria improve slowly and others where mechanical normalization never occurs. In the latter it is legitimate to expect recurrent AF episodes. Furthermore, in these patients, even when free from AF relapses, the unsatisfactory atrial mechanical performance results in the lack of clinical benefit. Thus cardioversion has no favorable effect, for example, in preventing a possible process already in progress towards the clinical picture of heart failure. We still lack data that allow us to affirm the possibility of differentiated pharmacological protocols of secondary prevention of AF on the basis of the STE findings, i.e., based on whether or not the patient has resumed a satisfactory atrial function. Finally, we are waiting for drugs able to improve the function of the reservoir phase of the atrial mechanical cycle. Is this an unrealistic expectation?

\section{Conclusions}

In the present retrospective cohort study as many as $22.5 \%$ of patients with persistent or long-standing persistent AF did not achieve the normalization of their atrial STE profile, even though they had been converted to sinus rhythm on the ECG by means of ECV. This finding prompts the attending physician to careful surveillance, even by executing serial STE assessment in order to evaluate the evolution of this atrial hypofunction over time.

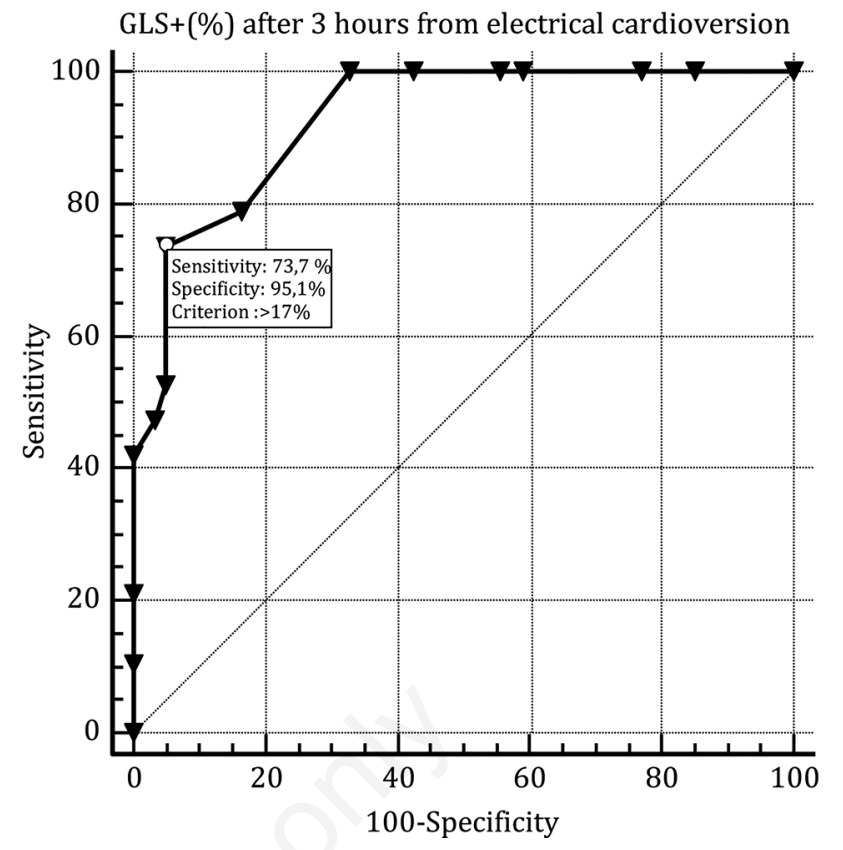

Figure 4. The value of "basal" global atrial strain (GSA+), that is the GSA+ measured after 3 hours from electrical cardioversion is associated with a significantly decreased risk of AF relapse over a 12-month follow-up when it is higher than to the cut-off value of $17 \%$.

\section{References}

1. Hwang J, Park HS, Jun SW, et al. The incidence of left atrial appendage thrombi on transesophageal echocardiography after pretreatment with apixaban for cardioversion in the real-world practice. PLoS One 2018;13:e0208734.

2. Dell'Era G, Rondano E, Franchi E, et al. Atrial asynchrony and function before and after electrical cardioversion for persistent atrial fibrillation. Eur J Echocardiogr 2010;11:577-83.

3. Vincenti A, Genovesi S, Sonaglioni A, et al. Mechanical atrial recovery after cardioversion in persistent atrial fibrillation evaluated by bidimensional speckle tracking echocardiography. J Cardiovasc Med (Hagerstown) 2019;20:745-51.

4. Pathan F, D'Elia N, Nolan MT, et al. normal ranges of left atrial strain by speckle-tracking echocardiography: A systematic review and meta-analysis. J Am Soc Echocardiogr 2017;30:5970.e8.

5. D'Andrea A, Vriz O, Ferrara F, et al. Reference ranges and physiologic variations of left E/e' ratio in healthy adults: Clinical and echocardiographic correlates. J Cardiovasc Echogr 2018;28:101-8.

6. Sonaglioni A, Vincenti A, Baravelli M, et al. Prognostic value of global left atrial peak strain in patients with acute ischemic stroke and no evidence of atrial fibrillation. Int $\mathrm{J}$ Cardiovasc Imaging 2019;35:603-13.

7. De Vecchis R, Paccone A, Di Maio M. Secondary prevention of nonvalvular atrial fibrillation: A retrospective cohort study. Cardiol Res 2019;10:223-9.

8. Lang RM, Badano LP, Mor-Avi V, et al. Recommendations for cardiac chamber quantification by echocardiography in adults: 
an update from the American Society of Echocardiography and the European Association of Cardiovascular Imaging. Eur Heart J Cardiovasc Imaging 2015;16:233-70.

9. Cianciulli TF, Saccheri MC, Lax JA, et al. Two-dimensional speckle tracking echocardiography for the assessment of atrial function. World J Cardiol 2010;2:163-70.

10. Zafrir B, Lund LH, Laroche C, et al. Prognostic implications of atrial fibrillation in heart failure with reduced, mid-range, and preserved ejection fraction: a report from 14,964 patients in the European Society of Cardiology Heart Failure LongTerm Registry. Eur Heart J 2018;39:4277-84.
11. Packer DL, Mark DB, Robb RA, et al. Effect of catheter ablation vs antiarrhythmic drug therapy on mortality, stroke, bleeding, and cardiac arrest among patients with atrial fibrillation: The CABANA randomized clinical trial. JAMA 2019;321:1261-74.

12. Manning WJ, Silverman DI, Katz SE, et al. Impaired left atrial mechanical function after cardioversion: relation to the duration of atrial fibrillation. J Am Coll Cardiol 1994;23:1535-40.

13. Manning WJ, Silverman DI, Katz SE, et al. Temporal dependence of the return of atrial mechanical function on the mode of cardioversion of atrial fibrillation to sinus rhythm. Am J Cardiol 1995;75:624-6. 